\title{
Not All Pulmonary Nodules in Smokers are Lung Cancer
}

\author{
Bhagwan Dass, MD; Jared Phillips, DO; Erin Jacobs, MD; and Jose E. Roman, MD
}

Diagnosis of pulmonary nodules requires an in-depth workup, including clinical evaluation, laboratory and pulmonary functions tests, and imaging, which helped to identify in this patient pulmonary rheumatoid arthritis, an important factor in patient mortality.

latsinats dentification of pulmonary nodules in older adults who smoke immediately brings concern for malignancy in the mind of clinicians. This is particularly the case in patients with significant smoking history. According to the National Cancer Institute in 2019, 12.9\% of all new cancer cases were lung cancers. ${ }^{1}$ Screening for lung cancer, especially in patients with increased risk from smoking, is imperative to early detection and treatment. However, $20 \%$ of patients will be overdiagnosed by lung cancer-screening techniques. ${ }^{2}$ The rate of malignancy noted on a patient's first screening computed tomography (CT) scan was between $3.7 \%$ and $5.5 \% .^{3}$

Rheumatoid arthritis (RA) is an autoimmune inflammatory condition that mainly affects the joints. Extraarticular manifestations can arise in various locations throughout the body, however. These manifestations are commonly observed in the skin, heart, and lungs. ${ }^{4}$ Prevalence of pulmonary rheumatoid nodules ranges from $<0.4 \%$ in radiologic studies to $32 \%$ in lung biopsies of patients with RA and nodules. ${ }^{5}$

Furthermore, there is a strong association between the risk of rheumatoid nodules in patients with positive serum rheumatoid factor (RF) and smoking history. ${ }^{6}$ Solitary pulmonary nodules in patients with RA can coexist with bronchogenic carcinoma, making their diagnosis more important. ${ }^{7}$

\section{CASE PRESENTATION}

A 54-year-old woman with a 30 pack-year smoking history and history of RA initially presented to the emergency department for cough and dyspnea for 5-day duration. Her initial diagnosis was bronchitis based on presenting symptom profile. A chest CT demonstrated 3 cavitary pulmonary nodules,
1 measuring $2.4 \times 2.0 \mathrm{~cm}$ in the right middle lobe, and 2 additional nodules, measuring $1.8 \times 1.4$ and $1.5 \times 1.4$ in the left upper lobe (Figure). She had no improvement of symptoms after a 7-day course of doxycycline. The patient was taking methotrexate $15 \mathrm{mg}$ weekly and golimumab $50 \mathrm{mg}$ subcutaneously every 4 weeks as treatment for RA, prescribed by her rheumatologist.

Pulmonology was consulted and a positron emission tomography-CT (PET-CT) confirmed several cavitary pulmonary nodules involving both lungs with no suspicious fluorodeoxyglucose (FDG) uptake. The largest lesion was in the right middle lobe with FDG uptake of 1.9. Additional nodules were found in the left upper lobe, measuring $1.8 \times 1.4 \mathrm{~cm}$ with FDG of 4.01, and in the left lung apex, measuring $1.5 \times 1.4 \mathrm{~cm}$ with uptake of 3.53 . CTguided percutaneous fine needle aspiration (PFNA) of the right middle lobe lung nodule demonstrated granuloma with central inflammatory debris. Grocott methenamine silver (GMS) stain was negative for fungal organism, acid-fast bacteria (AFB) stain was negative for acid-fast bacilli, and CD20 and CD3 immunostaining demonstrated mixed B- and T-cell populations. There was no evidence of atypia or malignancy. The biopsy demonstrated granuloma with central inflammatory debris on a background of densely fibrotic tissue and lympho-plasmatic inflammation. This finding confirmed the diagnosis of RA with pulmonary involvement.

Outpatient follow-up was established with a pulmonologist and rheumatologist. Methotrexate $15 \mathrm{mg}$ weekly and golimumab subcutaneously $50 \mathrm{mg}$ every 4 weeks were prescribed for the patient. The nodules are being monitored based on Fleischer guidelines with CT imaging 3 to 6 months
Author affiliations can be found at the end of this article.

Correspondence:

Bhagwan Dass

(bhagwan.dass.ctr@mail.mil)

Fed Pract. 2021;38(12). Published December 20. doi:10.12788/fp.0210 


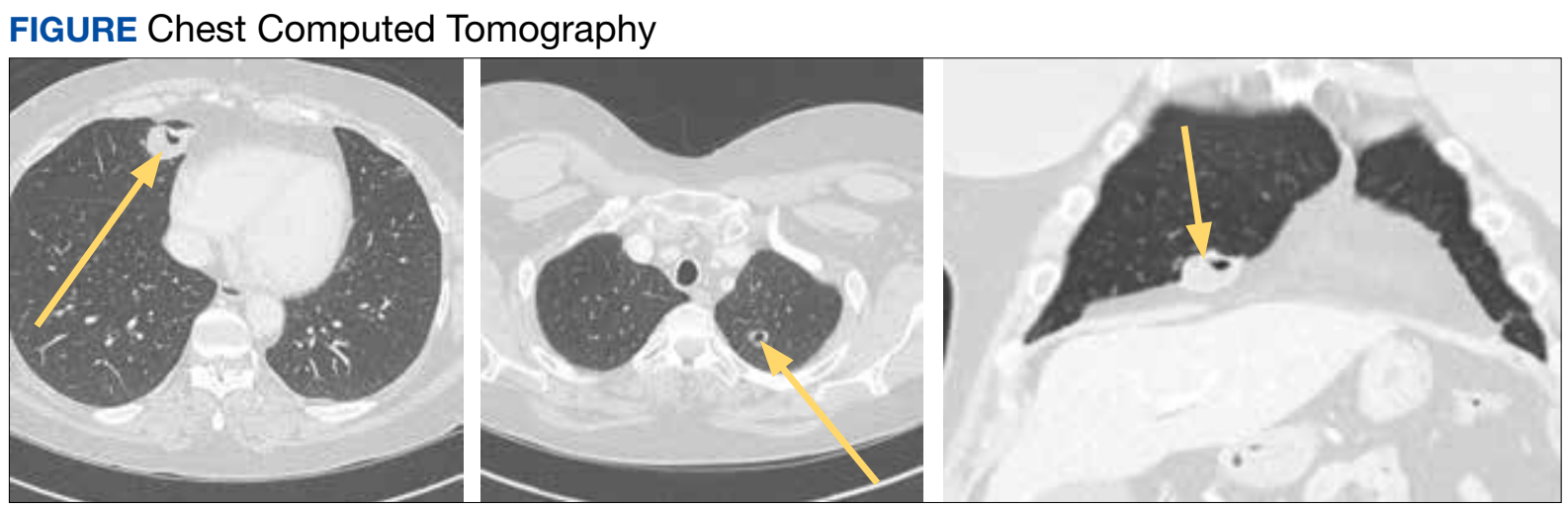

Arrows indicate cavitary rheumatoid nodules.

TABLE Patient Laboratory Test Results

\begin{tabular}{lc} 
Tests & Results \\
\hline Nuclear antibody & Negative \\
\hline Rheumatoid factor & 46 (range 0-15) \\
\hline Histoplasma antibody & Negative \\
\hline Coccidiodes antibody & Negative \\
\hline Quantiferon tuberculosis & Negative \\
\hline Acid-fast bacilli & Negative
\end{tabular}

following initial presentation. Further imaging will be considered at 18 to 24 months as well to further assess stability of the nodules and monitor for changes in size, shape, and necrosis. The patient also was encouraged to quit smoking. Her clinical course since the diagnosis has been stable.

\section{DISCUSSION}

The differential diagnosis for new multiple pulmonary nodules on imaging studies is broad and includes infectious processes, such as tuberculosis, as well as other mycobacterial, fungal, and bacterial infections. Noninfectious causes of lung disease are an even broader category of consideration. Noninfectious pulmonary nodules differential includes sarcoidosis, granulomatous with polyangiitis, hypersensitivity pneumonitis, methotrexate drug reaction, pulmonary manifestations of systemic conditions, such as RA chronic granulomatous disease and malignancy. ${ }^{8}$ Bronchogenic carcinoma was suspected in this patient due to her smoking history. Squamous cell carcinoma was also considered as the lesion was cavitary. AFB and GMS stains were negative for fungi. Langerhans cell histiocytosis were considered but ruled out as these lesions contain larger numbers of eosinophils than described in the pathology report. Histoplasma and coccidiosis laboratory tests were obtained as the patient lived in a region endemic to both these fungi but were negative (Table). A diagnosis of rheumatoid nodule was made based on the clinical setting, typical radiographic, histopathology features, and negative cultures.

This case is unique due to the quality and location of the rheumatoid nodules within the lungs. Pulmonary manifestations of RA are usually subcutaneous or subpleural, solid, and peripherally located. ${ }^{9}$ This patient's nodules were necrobiotic and located within the lung parenchyma. There was significant cavitation. These factors are atypical features of pulmonary RA.

Pulmonary RA can have many associated symptoms and remains an important factor in patient mortality. Estimates demonstrate that 10 to $20 \%$ of RA-related deaths are secondary to pulmonary manifestations. ${ }^{10}$ There are a wide array of symptoms and presentations to be aware of clinically. These symptoms are often nondescript, widely sensitive to many disease processes, and nonspecific to pulmonary RA. These symptoms include dyspnea, wheezing, and nonproductive cough. ${ }^{10}$ Bronchiectasis is a common symptom as well as small airway obstruction. ${ }^{10}$ Consolidated necrobiotic lesions are present in up to $20 \%$ of pulmonary RA cases. ${ }^{10}$ Generally these lesions are asymptomatic but can also be associated with pneumothorax, hemoptysis, and airway obstruction. ${ }^{10}$ Awareness of these symptoms is important for diagnosis and monitoring clinical improvement in patients. 
Further workup is necessary to differentiate malignancy-related pulmonary nodules and other causes; if the index of suspicion is high for malignancy as in our case, the workup should be more aggressive. Biopsy is mandatory in such cases to rule out infections and malignancy, as it is highly sensitive and specific. The main problem hindering management is when a clinician fails to include this in their differential diagnosis. This further elucidates the importance of awareness of this diagnosis. Suspicious lesions in a proper clinical setting should be followed up by imaging studies and confirmatory histopathological diagnosis. Typical follow-up is 3 months after initial presentation to assess stability and possibly 18 to 24 months as well based on Fleischer guidelines.

Various treatment modalities have been tried as per literature, including tocilizumab and rituximab. ${ }^{11,12}$ Our patient is currently being treated with golimumab based on outpatient rheumatologist recommendations.

\section{CONCLUSIONS}

This case demonstrates the importance of a careful workup to narrow a broad differential. Medical diagnosis of pulmonary nodules requires an in-depth workup, including clinical evaluation, laboratory and pulmonary functions tests, as well as various imaging studies.

\section{Author affiliations}

Bhagwan Dass is a Nephrologist, and Jose Roman is a Pulmonologist, both at Eglin Air Force Base (AFB) in Florida. Jared Phillips is a Family Medicine Resident PGY-3 at Eglin AFB. Erin Jacobs is a Fourth-Year Medical Student at the Uniformed Services University of the Health Sciences in Bethesda, Maryland.

\section{Author disclosures}

The authors report no actual or potential conflicts of interest or outside sources of funding with regard to this article.

\section{Disclaimer}

The opinions expressed herein are those of the authors and do not necessarily reflect those of Federal Practitioner, Frontline
Medical Communications Inc., the US Government, or any of its agencies. This article may discuss unlabeled or investigational use of certain drugs. Please review the complete prescribing information for specific drugs or drug combinations -including indications, contraindications, warnings, and adverse effects-before administering pharmacologic therapy to patients.

\section{Ethics and consent}

The authors report that this patient provided informed consent.

\section{References}

1. Lung and Bronchus Cancer - Cancer Stat Facts. SEER. Accessed February 2, 2020. https://seer.cancer.gov /statfacts/html/lungb.html

2. Shaughnessy AF. One in Five Patients Overdiagnosed with Lung Cancer Screening. Am Fam Physician. 2014 Jul 15;90(2):112.

3. McWilliams A, Tammemagi MC, Mayo JR, et al. Probability of cancer in pulmonary nodules detected on first screening CT. N Engl J Med. 2013;369;910-919. doi:10.1056/NEJMoa1214726

4. Stamp LK, Cleland LG. Rheumatoid arthritis. In: Thompson LU, Ward WE, eds. Optimizing Women's Health through Nutrition. CRC Press; 2008; 279-320.

5. Yousem SA, Colby TV, Carrington CB. Lung biopsy in rheumatoid arthritis. Am Rev Respir Dis. 1985;131(5):770-777. doi:10.1164/arrd.1985.131.5.770

6. Nyhäll-Wåhlin BM, Jacobsson LT, Petersson IF, Turesson C; BARFOT study group. Smoking is a strong risk factor for rheumatoid nodules in early rheumatoid arthritis. Ann Rheum Dis. 2006;65(5):601-606. doi:10.1136/ard.2005.039172

7. Shenberger KN, Schned AR, Taylor TH. Rheumatoid disease and bronchogenic carcinoma-case report and review of the literature. J Rheumatol. 1984;11:226-228.

8. Mukhopadhyay S, Wilcox BE, Myers JL, et al. Pulmonary necrotizing granulomas of unknown cause clinical and pathologic analysis of 131 patients with completely resected nodules. Chest. 2013;144(3):813-824. doi:10.1378/chest.12-2113

9. Ohshimo S, Guzman J, Costabel U, Bonella F. Differential diagnosis of granulomatous lung disease: clues and pitfalls: Number 4 in the Series "Pathology for the clinician." Edited by Peter Dorfmüller and Alberto Cavazza. Eur Respir Rev. 2017;26(145):170012. Published 2017 Aug 9. doi:10.1183/16000617.0012-2017

10. Brown KK. Rheumatoid lung disease. Proc Am Thorac Soc. 2007;4(5):443-448. doi:10.1513/pats.200703-045MS

11. Braun MG, Wagener P. Regression von peripheren und pulmonalen Rheumaknoten unter Rituximab-Therapie [Regression of peripheral and pulmonary rheumatoid nodules under therapy with rituximab]. Z Rheumatol. 2013;72(2):166-171. doi:10.1007/s00393-012-1054-0

12. Andres M, Vela P, Romera C. Marked improvement of lung rheumatoid nodules after treatment with tocilizumab. Rheumatology (Oxford). 2012;51(6):1132-1134. doi:10.1093/rheumatology/ker455 